\title{
Supratentorial giant cell ependymoma with extensive tumour cell vacuolization and calcification
}

\author{
Victor Willian Soares de Oliveira ${ }^{1}$, Fernanda Costa Nunes ${ }^{1,2}$, Mariana Canadas Linder ${ }^{1,2}$, Willian Baía ${ }^{3}$, Marcelo Neves Linhares ${ }^{3}$ and Jean \\ Costa Nunes ${ }^{1,2 *}$ \\ ${ }^{1}$ Division of Neuropathology, Universidade Federal de Santa Catarina (UFSC), Florianópolis, Santa Catarina (SC), Brazil \\ ${ }^{2}$ Neurodiagnostic Brasil - Diagnósticos em Neuropatologia, Florianópolis, SC, Brazil. \\ ${ }^{3}$ Division of Neurosurgery, Hospital Governador Celso Ramos, Florianópolis, SC, Brazil
}

\begin{abstract}
Objective: Ependymomas are rare brain tumours representing approximately 3\% of all intra-cerebral and spinal neoplasms. We describe a patient with a supratentorial ependymoma with wide calcification, exhibiting an undescribed association between giant cell and extensive tumour cell vacuolization morphological patterns.

Clinical Presentation: A 20-year-old woman presented with recurrent seizures. MRI showed a large, intra-axial tumour situated in the precuneal area of the occipital lobe. Histopathological assessment showed a heterogeneous well-delineated tumour, composed of cells with monomorphous ovoid nuclei and fine punctate chromatin. Pseudorosettes with tumour cells arranged radially around blood vessels were also observed in restricted areas. The tumour revealed wide areas with large, bizarre, often multinucleated cells with intracytoplasmic vacuoles of variable sizes. The tumour cells displayed glial fibrillary acid protein (GFAP) immunostaining, highlighting the perivascular pseudorosette cell processes. Epithelial membrane antigen (EMA) immunostaining revealed innumerous ring-like and dot-like reactions in classic, giant and vacuolized tumour cells.
\end{abstract}

Conclusion: This is the first report of a supratentorial ependymoma with giant cells, extensive tumour cell vacuolization and calcification. Additional studies are needed to determine the prognostic value of these features.

\section{Introduction}

Ependymomas are rare brain tumour representing approximately $3 \%$ of all intra-cerebral and spinal neoplasms [1]. Although the World Health Organization (WHO) classification recognizes three different subtypes of ependymoma, other rare variants have been described. We describe a patient with a supratentorial ependymoma with wide calcification, exhibiting an undescribed association between giant cell and extensive tumor cell vacuolization morphological patterns.

\section{Case report}

A 20-year-old woman with an unremarkable medical history presented with recurrent focal seizures during a period of two months. The patient reported visual aura with scotomas localized in her field of vision on the right side followed by hemiparesthesia of her right arm and loss of consciousness. Clinical examination showed superior homonymous quadrantanopsia in her field of vision on the right side. Computerized tomography and magnetic resonance imaging (MRI) (1.5 T) showed a large, intra-axial, expansive tumour $(3.0 \times 3.0 \times 2.7 \mathrm{~cm})$ located in the precuneus area of the left occipital lobe (Figure 1A). The tumour revealed regular boundaries, and no gadolinium enhancement was observed. Compressions of the corpus callosum and occipital horn of the left lateral ventricle were also detected. She underwent an occipital craniotomy, and the neoplasm was fully resected. At the macroscopic evaluation, dense areas with calcification were observed and confirmed by microscopic analysis. Histopathological assessment showed a heterogeneous well-delineated tumour composed of cells with monomorphous ovoid nuclei and fine punctate chromatin. Pseudorosettes with tumour cells arranged radially around blood vessels with anuclear zones were also observed in some areas (Figure 1B). Extensive perivascular and intratumoral calcification was detected (Figure 1C). The tumour revealed wide areas with large, bizarre, often multinucleated cells (Figure 1D). Some giant cells presented intracytoplasmic vacuoles of variable sizes (Figure 1E). Vimentin and S-100 protein demonstrated immunoreactivity in almost all tumour cells. The tumour cells displayed glial fibrillary acid protein (GFAP) immunostaining, highlighting the perivascular pseudorosette cell processes (Figure 1F and 1G). Some neoplastic cells demonstrated immunohistochemical positivity for microtubule-associated protein 2 (MAP2). Epithelial membrane antigen (EMA) immunostaining revealed numerous ring-like and dot-like reactions in classic, giant and vacuolized tumour cells (Figure $1 \mathrm{H}$ ). The Ki-67 labelling index was less than 2\%. Expression of p53 protein, IDH-1, and neuronal proteins such as synaptophysin and enolase was not observed. Approval by the Research Ethics Committee and informed consent were obtained.

${ }^{\star}$ Correspondence to: Jean Costa Nunes, Division of Neuropathology, Pathological Anatomy Service, 1st floor, Polydoro Ernani University Hospital of São Thiago, Federal University of Santa Catarina (UFSC), Rua Professor Maria Flora Pausewang, Trindade, Florianópolis, Santa Catarina (SC), Brazil, E-mail: jeanmedic@hotmail.com

Key words: brain neoplasms, neuroectodermal tumours, ependymoma, giant cells, vacuolization

Received: August 31, 2018; Accepted: September 11, 2018; Published: September 14, 2018 

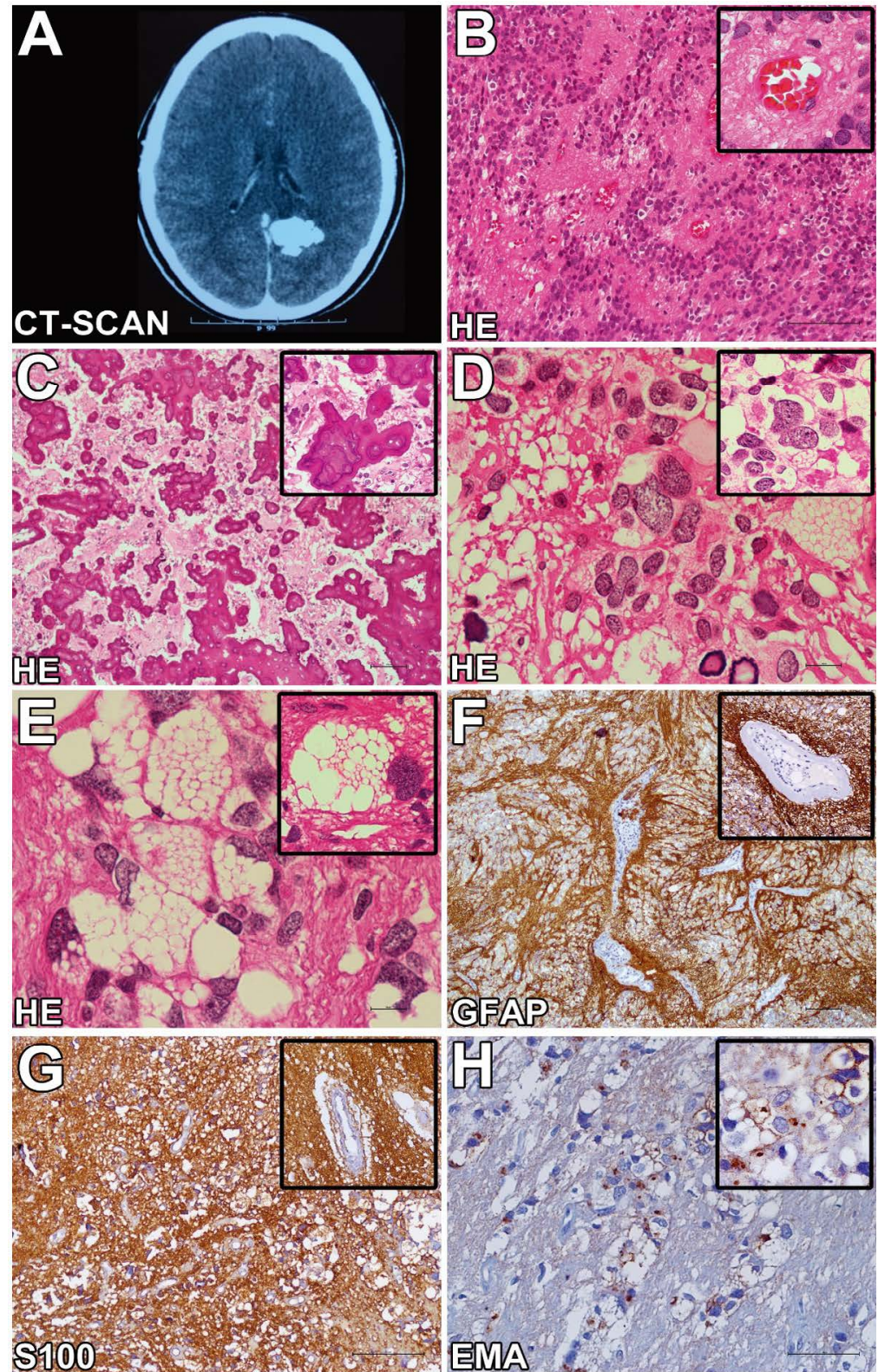

Figure 1. (A) CT-Scan showing. (B) Classical findings of ependymoma, composed of cells with monomorphous ovoid nuclei, fine punctate chromatin and pseudorosettes with tumour cells arranged radially around blood vessels with anuclear zones (scale bar $50 \mu \mathrm{m}$ ). (C) Extensive intratumoral and perivascular calcification (scale bar $100 \mu \mathrm{m})$. (D) Areas with large, bizarre, often multinucleated neoplastic cells (scale bar $50 \mu \mathrm{m}$ ). (E) Tumour cells with extensive intracytoplasmic vacuoles (scale bar $50 \mu \mathrm{m}$ ). (F) GFAP immunohistochemistry highlights the perivascular pseudorosette cell processes (scale bar $100 \mu \mathrm{m}$ ). (G) S100 protein immunoreactivity in neoplastic areas (scale bar $100 \mu \mathrm{m}$ ). (H) Epithelial membrane antigen (EMA) immunostaining revealed innumerous ring-like and dot-like reactions in classic, giant and vacuolized tumour cells (scale bar $50 \mu \mathrm{m})$

\section{Discussion}

According to the WHO classification, the ependymal tumours comprise a histologically heterogeneous group of tumors that include subependymoma (WHO grade I), myxopapillary ependymoma (WHO grade I), ependymoma (WHO grade II) and anaplastic ependymoma (WHO grade III) [2]. However, the WHO classification also indicates various other rare histological variants under the term "other patterns" [1]. The current analysis showed very rare morphostructural findings and revealed an undescribed association between two of these extremely rare variants. To exclude neoplasias with similar symptoms and histopathological findings, all brain tumours, including longterm epilepsy-associated tumors (LEAT) [3], that present bizarre and multinucleated giant cells were included in the differential diagnosis.
Pleomorphic xanthoastrocytoma (PXA) is an astrocytic neoplasm typically encountered in children and young adults, often located in the cerebral hemispheres [2]. Histopathologically, PXAs are characterized by cellular pleomorphism and xanthomatous changes [3]. Typical tumour cells in PXAs are large astroglial cells with abundant eosinophilic cytoplasm and large, often bizarre and sometimes multilobulated nuclei [3]. Based upon the histopathological features, this tumour was considered in our differential diagnosis. Despite immunoreactivity to GFAP and S-100 protein, other relevant characteristic features of this tumour, such as immunoreactivity to CD34 and a prominent reticulin network demonstrated with silver impregnation, were not detected. Giant cell glioblastoma (WHO grade IV) was also considered. A low proliferative index without geographic or pseudopalisading necrosis, 
microvascular proliferation and TP53 mutation ruled out this hypothesis. Subependymal giant cell astrocytoma (SEGA) is almost exclusively associated with tuberous sclerosis (TS) and located around the lateral ventricles [4]. The criteria for TS were not met; moreover, typical dot-like and ring-like EMA immunoreactivities were not described in this tumour, making this possibility unlikely. Angiocentric glioma (AG) consists of monomorphic, bipolar spindled cells with elongated nuclei and characteristic angiocentric arrangements around cortical blood vessels, forming perivascular pseudorosettes with an ependymoma-like appearance [3]. However, the immunoprofile can be similar to that of an ependymoma, and neither giant cells nor extensive tumour cell vacuolization have been previously recognized in this neoplasm. Gangliogliomas (WHO grade I) are one of the more common tumors in epilepsy surgical series [3]. They contain a combination of neoplastic glial cells and irregular groups of large, multipolar neurons with dysplastic features. In our case, dysplastic neurons with peri-membranous immunoreactivity to synaptophysin and CD34-positive multipolar cells were not detected, excluding this differential diagnosis.

Hirato J. et al. investigated ependymal tumour cells with extensive vacuolization [5]. Ultrastructurally, the neoplastic cells showed many intracytoplasmic lumina (ICL) with and without microvilli [5]. They considered that ICL without microvilli originated from several types of intracytoplasmic membrane-bounded organelles, including rough endoplasmic reticulum (rER), smooth ER and mitochondria [5]. Intracytoplasmic vacuolization of ependymal cells has been experimentally observed in the hypoxic state [6]. The etiopathogenesis involved in these unusual morphological features remains a matter of speculation.

The pleomorphism among giant cell ependymomas has been attributed to degenerative changes with no worse prognosis in the absence of other anaplastic features [1], although other authors interpreted the giant cells as a potential sign of a more aggressive clinical behavior [7].
The biological behavior of this tumor is difficult to predict in the present case. Our patient will be followed up for several years, but at this time, signs of recurrence have not been observed. For a better prognosis, our findings suggest a complete surgical resection and the absence of anaplastic features such as increased cellularity, brisk mitotic activity, microvascular proliferation and necrosis.

To the best of our knowledge, this is the first report of a supratentorial ependymoma with giant cells, extensive tumour cell vacuolization and calcification. Additional studies are needed to determine the prognostic value of these features and to elucidate whether they are entity-defining criteria or may just represent a spectrum of differentiation.

\section{Conflict of interest}

None declared.

\section{References}

1. Gessi M, Kuchelmeister K, Lauriola L, Pietsch T (2011) Rare histological variants in ependymomas: histopathological analysis of 13 cases. Virchows Archiv 459: 423-429.

2. Louis DN, Ohgaki H, Wiestler OD, Cavenee WK, Burger PC, et al. (2007) The 2007 WHO classification of tumours of the central nervous system. Acta neuropathologica 114: 97-109.

3. Thom M, Blumcke I, Aronica E (2012) Long-term epilepsy-associated tumors. Brain pathology 22: 350-379.

4. Li JY, Lopez JI, Powell SZ, Coons SW, Fuller GN (2012) Giant cell ependymomareport of three cases and review of the literature. Int J Clin Exp Pathol 5: 458-462.

5. Hirato J, Nakazato Y, Iijima M, Yokoo H, Sasaki A, et al. (1997) An unusual variant of ependymoma with extensive tumor cell vacuolization. Acta neuropathologica 93: 310-316.

6. Dodson RF, Tagashira Y, Chu LW (1975) Morphological studies of the ventricular wall in cerebral infarction. Experimental and Molecular Pathology 22: 294-301.

7. Jeon YK, Jung HW, Park SH (2004) Infratentorial giant cell ependymoma: a rare variant of ependymoma. Pathology-Research and Practice 200: 717-725.

Copyright: (C2018 Soares de Oliveira VW. This is an open-access article distributed under the terms of the Creative Commons Attribution License, which permits unrestricted use, distribution, and reproduction in any medium, provided the original author and source are credited. 